\title{
Block Spin Approach to the Singularity Properties of the Continued Fractions
}

\author{
Kumiko Hattori ${ }^{1}$, Tetsuya Hattori ${ }^{2}$ and Hiroshi Watanabe ${ }^{3}$ \\ 1 Department of Mathematics, Faculty of Science, University of Tokyo, Bunkyo-ku, Tokyo 113, Japan \\ ${ }^{2}$ Department of Physics, Faculty of Science, Gakushuuin University, Toshima-ku, Tokyo 171, Japan \\ ${ }^{3}$ Department of Mathematics, Faculty of Science, Tokyo Metropolitan University, Setagaya-ku, \\ Tokyo 158, Japan
}

\begin{abstract}
The massless singularity of a ferromagnetic Gaussian measure on $\mathbb{Z}_{+}$is studied by means of the coarse graining renormalization group method. The result gives information about a singularity behavior of a continued fraction and a time decay rate of a diffusion (random walk) on $\mathbb{Z}_{+}$.
\end{abstract}

\section{Introduction: Problem and Results}

We regard $\mathbb{R}^{\mathbb{Z}_{+}}$as a measurable space with the $\sigma$-algebra generated by the cylinder subsets of $\mathbb{R}^{\mathbb{Z}_{+}}$. Let us introduce the notion of ferromagnetic Gaussian measures on $\mathbb{R}^{\mathbb{Z}_{+}}$. For bounded positive sequences $J=\left(J_{n}\right)_{n \in \mathbb{Z}_{+}}$and $g=\left(g_{n}\right)_{n \in \mathbb{Z}_{+}}$satisfying

$$
\inf _{n \geqq 0} g_{n}>0,
$$

the pair $(J, g)$ is called a ferromagnetic pair. We define, for a ferromagnetic pair $(J, g)$, matrices $H(J)$ and $D(g)$ by putting, for $n, m \in \mathbb{Z}_{+}$,

$$
\begin{aligned}
H_{n m}(J) & =0, \quad|n-m|>2, \\
& =J_{n \wedge m}, \quad|n-m|=1, \\
& =-J_{n-1}-J_{n}, \quad n=m,
\end{aligned}
$$

and

$$
D_{n m}(g)=\delta_{n m} g_{n},
$$

where $n \wedge m=\min (n, m)$ and $J_{-1}=0$. The matrix $D(g)-H(J)$ induces a bounded linear operator on $l^{\infty}\left(\mathbb{Z}_{+}\right)=\left\{\left(\phi_{n}\right)_{n \in \mathbb{Z}_{+}}\left|\sup _{n \in \mathbb{Z}_{+}}\right| \phi_{n} \mid<\infty\right\}$ and it has a symmetric positive definite inverse (see Lemma 2.1 and 2.2). Then there exists a unique Gaussian probability measure $\mu_{J g}$ on $\mathbb{R}^{\mathbb{Z}_{+}}$with mean 0 and covariance $(D(g)-$ $H(J))^{-1}$. We refer to the probability measure $\mu_{J g}$ as the ferromagnetic Gaussian measure characterized by $(J, g)$ and write

$$
\langle F(\phi)\rangle(J, g)=\int F(\phi) \mu_{J g}(d \phi)
$$


for any integrable function $F(\phi)$ on $\mathbb{R}^{\mathbb{Z}}$. In particular,

$$
\left\langle\phi_{n} \phi_{m}\right\rangle(J, g)=(D(g)-H(J))_{n m}^{-1}, \quad n, m \in \mathbb{Z}_{+},
$$

holds. Our concern in this paper is the quantity:

$$
f(J, g)=\left\langle\phi_{0}^{2}\right\rangle(J, g) \text {. }
$$

Ferromagnetic Gaussian measures appear in the statistical mechanical theory of spin systems under the name "Gaussian model" or "free field" (for example, see [1]). In such a literature, $J_{n m}$ is called a ferromagnetic (nearest-neighbor) interaction, and $g_{n}$ corresponds to the square of mass. As is shown in Corollary 2.12, the function $f(J, t g)$ diverges as $t \downarrow 0$, where $(t g)_{n}=t g_{n}, n \in \mathbb{Z}_{+}$. The aim of this paper is to study the "massless singularity" of $f(J, t g)$ as $t \downarrow 0$. We show the following:

Theorem 1.1. If a ferromagnetic pair $(J, g)$ satisfies

$$
C_{1} n^{-\gamma} \leqq J_{n} \leqq C_{2} n^{-\gamma}, \quad n>0,
$$

for some constants $C_{1}, C_{2}>0$ and $\gamma \geqq 0$, then it holds that

$$
\lim _{t \downarrow 0} \frac{\log f(J, t g)}{\log t}=-\frac{\gamma+1}{\gamma+2}
$$

The theorem is restated in several ways. First we note that $f(J, t g)$ has an expression in the form of the continued fraction (see Appendix):

$$
f(J, t g)=\frac{1}{t g_{0}}+\frac{1}{J_{0}^{-1}}+\frac{1}{t g_{1}}+\frac{1}{J_{1}^{-1}}+\cdots
$$

Then we have:

Corollary 1.2. Let $\left(a_{n}\right)_{n \in \mathbb{Z}_{+}}$and $\left(b_{n}\right)_{n \in \mathbb{Z}_{+}}$be positive sequences such that

$$
\begin{aligned}
C_{3}<a_{n}<C_{4}, & n \geqq 1, \\
C_{3} n^{\gamma}<b_{n}<C_{4} n^{\gamma}, & n \geqq 1,
\end{aligned}
$$

for some constants $C_{3}, C_{4}>0$ and $\gamma \geqq 0$. Then, the continued fraction

$$
L(t)=\frac{1}{t a_{0}}+\frac{1}{b_{0}}+\frac{1}{t a_{1}}+\frac{1}{b_{1}}+\cdots, \quad t>0,
$$

satisfies

$$
\lim _{t \downarrow 0} \frac{\log L(t)}{\log t}=-\frac{\gamma+1}{\gamma+2}
$$

Secondly a ferromagnetic Gaussian measure can be related to the diffusion (random walk) problem on $\mathbb{Z}_{+}$. Consider the diffusion equation:

$$
\begin{aligned}
\frac{d}{d \tau} u(\tau) & =H(J) u(\tau), \quad \tau>0, \\
u_{n}(0) & =\delta_{0 n}, \quad n \in \mathbb{Z}_{+} .
\end{aligned}
$$

It is easily seen that the Laplace transform of $u_{n}(\tau)$ is given by the correlation 
function of a ferromagnetic Gaussian measure:

$$
\int_{0}^{\infty} u_{n}(\tau) e^{-\tau t} d \tau=\left\langle\phi_{0} \phi_{n}\right\rangle(J, t \mathbf{1}), \quad n \in \mathbb{Z}_{+},
$$

where $\mathbf{1}=(1,1, \ldots) \in l^{\infty}\left(\mathbb{Z}_{+}\right)$. Then, employing the Abelian theorem, we obtain the following corollary.

Corollary 1.3. Assume that the condition of the theorem is satisfied and that the solution of (1.9), (1.10) has the estimate

$$
C_{5} \tau^{-\tilde{D} / 2}<u_{0}(\tau)<C_{6} \tau^{-\tilde{D} / 2}, \quad \tau>1,
$$

for some $C_{5}, C_{6}>0$ and $\tilde{D}>0$. Then the exponent $\tilde{D}$ is given by

$$
\tilde{D}=\frac{2}{\gamma+2}
$$

The exponent $\tilde{D}$ is called the spectral dimension [3] (see also Definition of $\widetilde{d}(J, g)$ in Chap. 2.2).

The one dimensional diffusion problem has been extensively investigated by several authors in a general situation [4]. In particular, the fact stated in Theorem 1.1 may be obtained as a special case of the results of [5], where Krein's theory was used. We shall show the theorem by a quite different method, i.e. the coarse-graining renormalization group (block spin) method. Our analysis is an application of the renormalization group method for free fields on fractals studied in [6].

Our program is as follows. In Chap. 2, we shall show the well-definedness of the ferromagnetic Gaussian measure and prove some basic estimates. In Chap. 3, the coarse-graining renormalization for the Gaussian measure will be introduced. This plays the central role in Chap. 4 which is devoted to the proof of the main theorem.

Related problems are considered in $[7,8]$.

\section{Ferromagnetic Gaussian Measure}

In this chapter we show the well-definedness of the ferromagnetic Gaussian measure on one dimensional chain introduced in Chap. 1 and list basic properties that we use in the proof of Theorem 1.1.

\subsection{Well-Definedness of $\mu_{J g}$.}

Let $\mathbf{M}$ be the set of all real matrices $A=\left(A_{n m}\right)_{n, m \in \mathbb{Z}_{+}}$satisfying

$$
\|A\|=\sup _{n \in \mathbb{Z}_{+}} \sum_{m \in \mathbb{Z}_{+}}\left|A_{n m}\right|<\infty .
$$

Then $(\mathbf{M},\|\|)$ turns out to be a Banach algebra with the identity $I$ and acts on $l^{\infty}\left(\mathbb{Z}_{+}\right)$in the canonical sense.

Lemma 2.1. For a ferromagnetic pair $(J, g)$, we define $H(J)$ and $D(g)$ by $(1.2)$ and (1.3), respectively. Then $H(J)$ and $D(g)$ are in $\mathbf{M}$ and there exists a symmetric matrix 
$R(J, g) \in \mathbf{M}$ such that

$$
\begin{aligned}
& (D(g)-H(J)) R(J, g)=I, \\
& R(J, g)(D(g)-H(J))=I
\end{aligned}
$$

with the estimate

$$
\|R(J, g)\| \leqq\left(\sup _{n \in \mathbb{Z}_{+}} g_{n}\right)^{-1} .
$$

Proof. It is easily seen that $H(J), D(g) \in \mathbf{M}$. Let us show the existence of $R(J, g)$. Put

$$
\mu_{n}=g_{n}+J_{n-1}+J_{n}, \quad n \in \mathbb{Z}_{+} .
$$

We decompose $D(g)-H(J)$ as a sum of its diagonal part $D(\mu)$ and off-diagonal part $E(J)$ :

$$
D(g)-H(J)=D(\mu)-E(J)
$$

where

$$
\begin{aligned}
D(\mu)_{n m} & =0, \quad n \neq m, \\
& =\mu_{n}=g_{n}+J_{n-1}+J_{n}, \quad n=m, \\
E(J)_{n m} & =0, \quad|n-m| \neq 1, \\
& =J_{n \wedge m}, \quad|n-m|=1 .
\end{aligned}
$$

Then the Neumann series $\sum_{N=0}^{\infty}\left(D(\mu)^{-1} E(J)\right)^{N}$ converges in $\mathbf{M}$, and hence $D(g)$ $H(J)=D(\mu)\left(I-D(\mu)^{-1} E(J)\right)$ has the inverse $R(J, g)$ :

$$
R(J, g)=\sum_{N=0}^{\infty}\left(D(\mu)^{-1} E(J)\right)^{N} D(\mu)^{-1} .
$$

The symmetry of $R(J, g)$ is trivial. In order to show (2.4), we rewrite (2.3) as

$$
R(J, g) D(g)=I+R(J, g) H(J) .
$$

Put $\mathbf{1}={ }^{t}(1,1, \ldots) \in l^{\infty}\left(\mathbb{Z}_{+}\right)$. If we note that $H(J) \mathbf{1}=0$, we have

$$
R(J, g) D(g) \mathbf{1}=\mathbf{1} .
$$

This implies (2.4).

We now need a positive definiteness of $R(J, g)$.

Lemma 2.2. For $\xi \in l^{\infty}\left(\mathbb{Z}_{+}\right)$such that $\xi \neq 0$ and $\xi_{n} \neq 0$ only for finite n's, it holds that

$$
\langle\xi, R(J, g) \xi\rangle>0 \text {. }
$$

Proof. Put $\eta=R(J, g) \xi$. Then:

$$
\sum_{n \in \mathbb{Z}_{+}} \eta_{n}^{2}\langle\infty, \quad\langle\xi, R(J, g) \xi\rangle=\langle(D(g)-H(J)) \eta, \eta\rangle .
$$

If we note that

$$
\langle(D(g)-H(J)) \eta, \eta\rangle=\sum_{n \in \mathbb{Z}_{+}}\left[g_{n} \eta_{n}^{2}+J_{n}\left(\eta_{n}-\eta_{n+1}\right)^{2}\right],
$$


we have the lemma.

By the help of the above lemmas, the standard method of the probability theory ensures the existence of the ferromagnetic Gaussian measure $\mu_{J g}$ with mean 0 and covariance $R(J, g)$.

We now pick up some convenient formulas from the above argument. Let us prepare some notations. For $i, j \in \mathbb{Z}_{+}$, we say that a sequence $w=$ $\left(w_{0}, w_{1}, \ldots, w_{N}\right) \subset \mathbb{Z}_{+}$is a walk from $i$ to $j$ if $w_{0}=i, w_{N}=j$ and $\left|w_{k}-w_{k+1}\right|=1$, $0 \leqq k \leqq N-1$. The set of all walks from $i$ to $j$ is denoted by $W(i, j)$. For a walk $w=\left(w_{0}, w_{1}, \ldots, w_{N}\right) \in W(i, j)$ and a ferromagnetic pair $(J, g)$, we put

$$
\begin{aligned}
& J_{w}=\prod_{k=0}^{N-1} J_{w_{k} \wedge w_{k+1}}, \\
& \mu_{n}=g_{n}+J_{n}+J_{n-1}, \\
& \mu_{w}=\prod_{k=0}^{N} \mu_{w_{k}} .
\end{aligned}
$$

Proposition 2.3. The correlation function of a ferromagnetic Gaussian measure satisfies the following equalities:

$$
\begin{gathered}
\left\langle\phi_{i} \phi_{j}\right\rangle(J, g)=\sum_{w \in W(i, j)} J_{w} / \mu_{w}, \\
\sum_{j \in \mathbb{Z}_{+}}\left\langle\phi_{i} \phi_{j}\right\rangle(J, g) g_{j}=1 \\
\left\langle\phi_{i} \phi_{j}\right\rangle(c J, c g)=c^{-1}\left\langle\phi_{i} \phi_{j}\right\rangle(J, g),
\end{gathered}
$$

where $i, j \in \mathbb{Z}_{+}$and $c>0$.

Proof. The "random walk representation" (2.8) is equivalent to (2.5) and the formula (2.9) is nothing but (2.6). The last equality is trivial.

\subsection{Basic Properties.}

In the following, unless otherwise stated, $(J, g)$ and $\left(J^{\prime}, g^{\prime}\right)$ are arbitrary ferromagnetic pairs.

Definition. Consider the quantity $f(J, g)=\left\langle\phi_{0}^{2}\right\rangle(J, g)$. If the limit

$$
\lim _{t \downarrow 0} \frac{\log f(J, t g)}{\log t}
$$

exists, we say that the ferromagnetic pair $(J, g)$ has the spectral dimension, and we define $\tilde{d}(J, g)$, the spectral dimension of $(J, g)$, by,

$$
\tilde{d}(J, g) / 2-1=\lim _{t \downarrow 0} \frac{\log f(J, t g)}{\log t} .
$$

In the remainder of this chapter, we shall study the behavior of $f(J, g)$ and $\tilde{d}(J, g)$ under the change of the parameters $J$ and $g$. 
Lemma 2.4. For a ferromagnetic pair $(J, g)$,

$$
0<\left\langle\phi_{0} \phi_{n}\right\rangle(J, g) \leqq \frac{1}{(n+1) \inf _{m \in \mathbb{Z}_{+}} g_{m}}, \quad n \in \mathbb{Z}_{+} \cdot
$$

Proof. The positivity of $\left\langle\phi_{0} \phi_{n}\right\rangle(J, g)$ is trivial from the random walk representation (2.8):

$$
\left\langle\phi_{0} \phi_{n}\right\rangle(J, g)=\sum_{w \in W(0, n)} \frac{J_{w}}{\mu_{w}} .
$$

Since each term of the random walk representation is positive, we can make resummations and throw away terms to obtain lower bounds. We follow the method of stopping time arguments:

$$
\begin{aligned}
\left\langle\phi_{0} \phi_{n}\right\rangle(J, g) & =\sum_{w \in W(0, n)} \frac{J_{w}}{\mu_{w}}=\sum_{w^{\prime}}^{\prime} \frac{J_{w^{\prime}}}{\mu_{w^{\prime}}} \mu_{n+1} \sum_{w^{\prime \prime} \in W(n+1,0)} \frac{J_{w^{\prime \prime}}}{\mu_{w^{\prime \prime}}}+\sum_{w}^{\prime \prime} \frac{J_{w}}{\mu_{w}} \\
& >\frac{J_{n}}{\mu_{n}} \sum_{w^{\prime \prime} \in W(n+1,0)} \frac{J_{w^{\prime \prime}}}{\mu_{w^{\prime \prime}}}>\sum_{w^{\prime \prime} \in W(n+1,0)} \frac{J_{w^{\prime \prime}}}{\mu_{w^{\prime \prime}}} \\
& =\left\langle\phi_{0} \phi_{n+1}\right\rangle(J, g) .
\end{aligned}
$$

Here the summation $\sum^{\prime}$ is over all walks $w^{\prime}=(n, n+1)$ or $\left(n, j_{1}, j_{2}, \ldots, j_{m}, n+1\right)$ ( $m=1,2, \ldots)$ starting from $n$ and ending at $n+1$ with the property that $j_{k} \neq n+1$ for all $k \in\{1,2, \ldots, m\}$, and the summation $\sum^{\prime \prime}$ is over all walks $w=\left(n, j_{1}, j_{2}, \ldots, j_{m}, 0\right)$ (or $(n, 0)$ if $n=1$ ) starting from $n$ and ending at 0 with the property that $j_{k} \neq n+1$ for all $k \in\{1,2, \ldots, m\}$. In the calculation, we have also used the fact that

$$
\mu_{n}=g_{n}+J_{n}+J_{n-1}>J_{n} .
$$

Combining (2.12) with (2.9), we have

$$
\begin{aligned}
1 & \left.=\sum_{m=0}^{\infty} g_{m}\left\langle\phi_{0} \phi_{m}\right\rangle(J, g)\right\rangle\left(\inf _{m \in \mathbb{Z}_{+}} g_{m}\right) \sum_{m=0}^{n}\left\langle\phi_{0} \phi_{m}\right\rangle(J, g) \\
& >\left(\inf _{m \in \mathbb{Z}_{+}} g_{m}\right)(n+1)\left\langle\phi_{0} \phi_{n}\right\rangle(J, g) .
\end{aligned}
$$

Lemma 2.5. Let $(J, g)$ and $\left(J, g^{\prime}\right)$ be two ferromagnetic pairs. Define $g(s)=\left(g(s)_{n}\right)_{n \in \mathbb{Z}_{+}}$, $(0 \leqq s \leqq 1) b y$,

$$
g(s)_{n}=g_{n} s+g_{n}^{\prime}(1-s)
$$

Then,

$$
\frac{d}{d s} f(J, g(s))=-\sum_{n}\left(g_{n}-g_{n}^{\prime}\right)\left\langle\phi_{0} \phi_{n}\right\rangle(J, g(s))^{2} .
$$

Proof. From (2.2) and (2.3), we have, for $s, s^{\prime}>0$;

$$
\sum_{k \in \mathbb{Z}_{+}}(D(g(s))-H(J))_{n k}\left\langle\phi_{k} \phi_{0}\right\rangle(J, g(s))=\delta_{n, 0},
$$


and

$$
\begin{aligned}
& \sum_{n, k \in \mathbb{Z}_{+}}\left\langle\phi_{m} \phi_{n}\right\rangle\left(J, g\left(s^{\prime}\right)\right)\left(D\left(g\left(s^{\prime}\right)\right)-H(J)\right)_{n k}\left\langle\phi_{k} \phi_{0}\right\rangle(J, g(s)) \\
& \quad=\left\langle\phi_{m} \phi_{0}\right\rangle(J, g(s)) .
\end{aligned}
$$

Multiply (2.15) by $\left\langle\phi_{m} \phi_{n}\right\rangle\left(J, g\left(s^{\prime}\right)\right)$, sum over $n \in \mathbb{Z}_{+}$, and subtract (2.16) to obtain,

$$
\begin{aligned}
& \left\langle\phi_{m} \phi_{0}\right\rangle\left(J, g\left(s^{\prime}\right)\right)-\left\langle\phi_{m} \phi_{0}\right\rangle(J, g(s)) \\
& \quad=\sum_{n \in \mathbb{Z}_{+}}\left(g(s)-g\left(s^{\prime}\right)\right)_{n}\left\langle\phi_{m} \phi_{n}\right\rangle\left(J, g\left(s^{\prime}\right)\right)\left\langle\phi_{n} \phi_{0}\right\rangle(J, g(s)) \\
& =\sum_{n \in \mathbb{Z}_{+}}\left(g_{n}-g_{n}^{\prime}\right)\left(s-s^{\prime}\right)\left\langle\phi_{m} \phi_{n}\right\rangle\left(J, g\left(s^{\prime}\right)\right)\left\langle\phi_{n} \phi_{0}\right\rangle(J, g(s)) .
\end{aligned}
$$

From (2.17), we have the continuity of $\left\langle\phi_{m} \phi_{0}\right\rangle(J, g(s))$ with respect to $s$. If we put $m=0$ in (2.17), divide by $\left(s^{\prime}-s\right)$, and use the continuity of $\left\langle\phi_{m} \phi_{0}\right\rangle(J, g(s))$, we obtain the desired result.

Corollary 2.6. (i) If

$$
g_{n} \leqq g_{n}^{\prime}, \quad n \in \mathbb{Z}_{+},
$$

then

$$
f\left(J, g^{\prime}\right) \leqq f(J, g)
$$

(ii) If

$$
g_{n} \leqq g_{n}^{\prime}, \quad n \in \mathbb{Z}_{+},
$$

then

$$
\left|\log f(J, g)-\log f\left(J, g^{\prime}\right)\right| \leqq\left(\inf _{n} g_{n}\right)^{-2} f\left(J, g^{\prime}\right)^{-1} \sum_{n \in \mathbb{Z}_{+}}(n+1)^{-2}\left(g_{n}^{\prime}-g_{n}\right) .
$$

Proof. (i). From the assumption and Lemma 2.5, the statement follows directly. (ii). From Lemma 2.5, Lemma 2.4, and the assumption, we have,

$$
\left|\frac{d}{d s} f(J, g(s))\right| \leqq \sum_{n}\left(g_{n}^{\prime}-g_{n}\right)(n+1)^{-2}\left(\underset{n}{\inf g(s)_{n}}\right)^{-2} .
$$

Therefore, using the assumption and (i),

$$
\begin{aligned}
& \left|\log f(J, g)-\log f\left(J, g^{\prime}\right)\right|=\left|\int_{0}^{1} \frac{d}{d s} \log f(J, g(s)) d s\right| \\
& \leqq \int_{0}^{1} \frac{1}{f(J, g(s))} \sum_{n}\left(g_{n}^{\prime}-g_{n}\right)(n+1)^{-2}\left(\inf _{n} g(s)_{n}\right)^{-2} d s \\
& \leqq\left(\inf _{n} g_{n}\right)^{-2} f\left(J, g^{\prime}\right)^{-1} \sum_{n \in \mathbb{Z}_{+}}(n+1)^{-2}\left(g_{n}^{\prime}-g_{n}\right) .
\end{aligned}
$$

Corollory 2.7. If $\tilde{d}(J, g)$ exists, then $\tilde{d}\left(J, g^{\prime}\right)$ also exists, and $\tilde{d}(J, g)=\tilde{d}\left(J, g^{\prime}\right)$.

Proof. Since $g=\left(g_{n}\right)_{n \in \mathbb{Z}_{+}}$and $g^{\prime}=\left(g_{n}^{\prime}\right)_{n \in \mathbb{Z}_{+}}$are bounded positive sequences satisfying 
(1.1), there exist positive constants $M$ and $M^{\prime}$ such that

From (2.18),

$$
M g_{n}<g_{n}^{\prime}<M^{\prime} g_{n}, \text { for all } n \in \mathbb{Z}_{+} \text {. }
$$

$$
f\left(J, t M^{\prime} g\right) \leqq f\left(J, t g^{\prime}\right) \leqq f(J, t M g), \quad \text { for } \quad t>0 .
$$

Therefore if $0<t<1$,

$$
\frac{\log f\left(J, t M^{\prime} g\right)}{\log t} \geqq \frac{\log f\left(J, t g^{\prime}\right)}{\log t} \geqq \frac{\log f(J, t M g)}{\log t} .
$$

Clearly,

$$
\lim _{t \downarrow 0} \frac{\log f(J, t M g)}{\log t}=\lim _{t \downarrow 0} \frac{\log f(J, t g)}{\log t-\log M}=\lim _{t \downarrow 0} \frac{\log f(J, t g)}{\log t}=\frac{\tilde{d}(J, g)}{2}-1,
$$

from which the statement follows.

Corollary 2.7 shows that $\widetilde{d}(J, g)$ is independent of the choice of $g=\left(g_{n}\right)$. Henceforth we shall write

$$
\tilde{d}(J) \equiv \tilde{d}(J, g) .
$$

Lemma 2.8. Let $(J, g)$ and $\left(J^{\prime}, g\right)$ be two ferromagnetic pairs. Define $J(s)=\left(J(s)_{n}\right)_{n \in \mathbb{Z}_{+}}$, $(0 \leqq s \leqq 1) b y$,

Then,

$$
J(s)_{n}=J_{n} s+J_{n}^{\prime}(1-s)
$$

$$
\frac{d}{d s} f(J(s), g)=-\sum_{n}\left(J_{n}-J_{n}^{\prime}\right) \frac{1}{2}\left(\left\langle\phi_{0} \phi_{n}\right\rangle(J(s), g)-\left\langle\phi_{0} \phi_{n+1}\right\rangle(J(s), g)\right)^{2} .
$$

Proof. Direct application of the method used in the proof of Lemma 2.5 proves this lemma.

Corollary 2.9. (i) If

then

$$
J_{n} \leqq J_{n}^{\prime}, \quad n \in \mathbb{Z}_{+},
$$

(ii) If

$$
f\left(J^{\prime}, g\right) \leqq f(J, g)
$$

$$
J_{n} \leqq J_{n}^{\prime}, \quad n \in \mathbb{Z}_{+},
$$

then

$$
\left|\log f(J, g)-\log f\left(J^{\prime}, g\right)\right| \leqq 2^{-1}\left(\inf _{n} g_{n}\right)^{-2} f\left(J^{\prime}, g\right)^{-1} \sum_{n \in \mathbb{Z}_{+}}(n+1)^{-2}\left(J_{n}^{\prime}-J_{n}\right) .
$$

Proof. (i) From the assumption and Lemma 2.8, the statement follows directly. (ii). From Lemma 2.8, Lemma 2.4, and the assumption, we have,

$$
\left|\frac{d}{d s} f(J(s), g)\right| \leqq \sum_{n}\left(J_{n}^{\prime}-J_{n}\right) \frac{1}{2}\left(\left\langle\phi_{0} \phi_{n}\right\rangle(J(s), g)-\left\langle\phi_{0} \phi_{n+1}\right\rangle(J(s), g)\right)^{2}
$$




$$
\begin{aligned}
& \leqq \sum_{n}\left(J_{n}^{\prime}-J_{n}\right) \frac{1}{2}\left\langle\phi_{0} \phi_{n}\right\rangle(J(s), g)^{2} \\
& \leqq \frac{1}{2} \sum_{n}\left(J_{n}^{\prime}-J_{n}\right)(n+1)^{-2}\left(\inf _{n} g(s)_{n}\right)^{-2} .
\end{aligned}
$$

Therefore, using the assumption and (i),

$$
\begin{aligned}
& \left|\log f(J, g)-\log f\left(J^{\prime}, g\right)\right|=\left|\int_{0}^{1} \frac{d}{d s} \log f(J(s), g) d s\right| \\
& \leqq 2^{-1}\left(\inf _{n} g_{n}\right)^{-2} f\left(J^{\prime}, g\right)^{-1} \sum_{n \in \mathbb{Z}_{+}}(n+1)^{-2}\left(J_{n}^{\prime}-J_{n}\right) .
\end{aligned}
$$

Corollary 2.10. Assume that $\tilde{d}(J)$ exists. If there exist positive constants $C$ and $C^{\prime}$ which are independent of $n \in \mathbb{Z}_{+}$such that,

$$
C J_{n}<J_{n}^{\prime}<C^{\prime} J_{n}, \quad n \in \mathbb{Z}_{+},
$$

then $\tilde{d}\left(J^{\prime}\right)$ also exists, and

$$
\tilde{d}(J)=\tilde{d}\left(J^{\prime}\right)
$$

Proof. From (2.22),

$$
f(C J, t g) \leqq f\left(J^{\prime}, t g\right) \leqq f\left(C^{\prime} J, t g\right), \quad \text { for } \quad t>0 .
$$

Using (2.10), we have

$$
f\left(J, t C^{-1} g\right) / C \leqq f\left(J^{\prime}, t g\right) \leqq f\left(J, t C^{\prime-1} g\right) / C^{\prime} .
$$

The statement is now reduced to Corollary 2.7.

Lemma 2.11. Define a ferromagnetic pair $(J, g)$ by

$$
\begin{aligned}
& g_{0}=g^{*} / 2, \\
& g_{n}=g^{*}, \quad n=1,2,3, \ldots \\
& J_{n}=J^{*}, \quad n \in \mathbb{Z}_{+} \text {, }
\end{aligned}
$$

where $g^{*}$ and $J^{*}$ are positive constants. Then

$$
f(J, g)=\left(g^{*} J^{*}+g^{* 2} / 4\right)^{-1 / 2} .
$$

Proof. From (1.6) we see that $f(J, g)$ must satisfy,

$$
f(J, g)=\frac{1}{g^{*} / 2+X},
$$

where

$$
X=\frac{1}{J^{*-1}+\frac{1}{g^{*}+X}},
$$

from which we obtain

$$
X=-g^{*} / 2 \pm\left(J^{*} g^{*}+g^{* 2} / 4\right)^{1 / 2}
$$


If we put this into (2.25), we see that $f(J, g)$ must satisfy:

$$
f(J, g)= \pm\left(g^{*} J^{*}+g^{* 2} / 4\right)^{-1 / 2} .
$$

Since we already know that $f(J, g) R(J, g)_{00}$ exists and is positive, we have the statement.

\section{Corollary 2.12.}

$$
\lim _{t \downarrow 0} f(J, t g)=\infty .
$$

Proof. Since $J=\left(J_{n}\right)_{n \in \mathbb{Z}_{+}}$and $g=\left(g_{n}\right)_{n \in \mathbb{Z}_{+}}$are bounded sequences, there exists a constant $M(>0)$ such that

$$
J_{n}<M, \text { and } g_{n}<M, \text { for all } n \in \mathbb{Z}_{+} .
$$

Using (2.18), (2.22), and Lemma 2.11, we have

$$
f(J, t g) \geqq f(M, t M)=\left(t M^{2}+t^{2} M^{2} / 4\right)^{-1 / 2} \uparrow \infty, \quad \text { as } \quad t \downarrow 0 .
$$

\section{The Coarse Graining Method}

In Chap. 2, we defined the spectral dimension $\widetilde{d}(J)$ which describes the "massless singularity" of the measure $\mu_{J g}$, and derived some properties of $\tilde{d}(J)$, assuming its existence. In this chapter, we prove a simple lemma which gives us a sufficient condition for the existence of $\tilde{d}(J)$. In this lemma, we assume that $f(J, g)=$ $\left\langle\phi_{0}^{2}\right\rangle(J, g)$ satisfies an identity (in the massless limit) under the scale change of parameters $(J, g)$. To obtain the identity, we then consider a marginal distribution of $\mu_{J g}$, by "integrating" the variables $\phi_{2 n+1}, n \in \mathbb{Z}_{+}$. The intuition of this procedure came from the coarse graining renormalization group method, which appears in statistical mechanics.

Lemma 3.1. Consider a ferromagnetic pair $(J, g)$. If, there exist positive constants $\alpha$ and $\beta$ such that $\beta>\alpha$ and

$$
\lim _{t \downarrow 0} \frac{f(J, t g)}{f(\alpha J, \beta t g)}=1
$$

then $\tilde{d}(J)$ exists, and

$$
\tilde{d}(J)=\frac{2 \log \beta}{\log (\beta / \alpha)}
$$

Proof. Put

and define

$$
x=-(\log t) / \log (\beta / \alpha)
$$

$$
F(x)=\log \left\{f\left(J,(\alpha / \beta)^{x} g\right)\right\}+(\log \alpha) x .
$$

From the assumption and (2.10),

$$
\lim _{x \rightarrow \infty}\{F(x)-F(x-1)\}=0,
$$


from which we have

$$
\lim _{x \rightarrow \infty} \frac{F(x)}{x}=0 .
$$

From the definition of $x$ and $F(x)$ we have

$$
\lim _{t \downarrow 0} \frac{\log f(J, t g)}{\log t}=\frac{\log \alpha}{\log (\beta / \alpha)} .
$$

Proposition 3.2. Consider a ferromagnetic pair $(J, g)$. Define another ferromagnetic pair $(\tilde{J}, \tilde{g})$ by

$$
\begin{aligned}
& \tilde{J}_{n}=\frac{J_{2 n} J_{2 n+1}}{g_{2 n+1}+J_{2 n}+J_{2 n+1}}, \\
& \tilde{g}_{n}=g_{2 n}+\frac{J_{2 n+1}}{g_{2 n-1}+J_{2 n-2}+J_{2 n-1}} g_{2 n-1}+\frac{J_{2 n}}{g_{2 n+1}+J_{2 n}+J_{2 n+1}} g_{2 n+1}, \quad n \in \mathbb{Z}_{+} .
\end{aligned}
$$

Then

$$
f(J, g)=f(\tilde{J}, \tilde{g}) .
$$

Proof. The Gaussian probability measure $\mu_{J g}$ has mean 0 and covariance $R(J, g)=$ $(D(g)-H(J))^{-1}$. Consider a measurable map

$$
p: \mathbb{R}^{\mathbb{Z}+} \rightarrow \mathbb{R}^{\mathbb{Z}+}
$$

defined by;

$$
p:\left(\phi_{0}, \phi_{1}, \phi_{2}, \ldots\right) \mapsto\left(\phi_{0}, \phi_{2}, \phi_{4}, \ldots\right)
$$

The image measure

$$
\tilde{\mu}_{J g}=\mu_{J g} p^{-1}
$$

is again a Gaussian probability measure with mean 0 and covariance $\tilde{R}(J, g)=$ $\left(\tilde{R}(J, g)_{n m}\right)_{n, m \in \mathbb{Z}_{+}}$, where

$$
\tilde{R}(J, g)_{n m}=R(J, g)_{2 n, 2 m} .
$$

As in Lemma 2.1, we decompose $D(g)-H(J)$ into a sum of diagonal part $D(\mu)$ and off-diagonal part $E(J)$ :

where

$$
D(g)-H(J)=D(\mu)-E(J),
$$

$$
\begin{aligned}
D(\mu)_{n m} & =0, \quad n \neq m, \\
& =\mu_{n}=g_{n}+J_{n-1}+J_{n}, \quad n=m, \\
E(J)_{n m} & =0, \quad|n-m| \neq 1, \\
& =J_{n \wedge m}, \quad|n-m|=1 .
\end{aligned}
$$

As have been proved in Lemma 2.1,

$$
\tilde{R}(J, g)_{n m}=R(J, g)_{2 n, 2 m}=\sum_{N=0}^{\infty}\left\{\left(D(\mu)^{-1} E(J)\right)^{N} D(\mu)^{-1}\right\}_{2 n, 2 m} .
$$


On the other hand, from the definition of $D(\mu)$ and $E(J)$, we have, for odd $N$,

$$
\left\{\left(D(\mu)^{-1} E(J)\right)^{N} D(\mu)^{-1}\right\}_{2 n, 2 m}=0 .
$$

Therefore,

$$
\begin{aligned}
\tilde{R}(J, g)_{n m} & =\sum_{N=0}^{\infty}\left\{\left(D(\mu)^{-1} E(J)\right)^{2 N} D(\mu)^{-1}\right\}_{2 n, 2 m} \\
& =\sum_{N=0}^{\infty}\left\{\left(D(\mu)^{-1} E(J) D(\mu)^{-1} E(J)\right)^{N} D(\mu)^{-1}\right\}_{2 n, 2 m} .
\end{aligned}
$$

If we define $D_{0}, D_{1} \in \mathbf{M}$ by

$$
\begin{aligned}
D_{0, n m} & =0, \quad n \neq m, \\
& =D(\mu)_{2 n}, \quad n=m, \\
D_{1, n m} & =0, \quad|n-m| \neq 1, \\
& =\left(E(J) D(\mu)^{-1} E(J)\right)_{2 n, 2 m}, \quad|n-m|=1,
\end{aligned}
$$

we have

$$
\begin{aligned}
\sum_{N=0}^{\infty}\left\{\left(D(\mu)^{-1} E(J) D(\mu)^{-1} E(J)\right)^{N} D(\mu)^{-1}\right\}_{2 n, 2 m} & =\sum_{N=0}^{\infty}\left\{\left(D_{0}^{-1} D_{1}\right)^{N} D_{0}^{-1}\right\}_{n m} \\
& =\left(D_{0}-D_{1}\right)_{n m}^{-1},
\end{aligned}
$$

where the last equality can be proved in the same way as Lemma 2.1. If we write down the last expression explicitly, we find that it is equal to $R(\tilde{J}, \tilde{g})$. If we use (3.4) we obtain, in particular,

$$
f(J, g)=R(J, g)_{00}=\tilde{R}(J, g)_{00}=R(\tilde{J}, \tilde{g})_{00}=f(\tilde{J}, \tilde{g})
$$

\section{Proof of the Main Theorem}

Proof of Theorem 1.1. For $\gamma=0$, the theorem is a direct consequence of Lemma 2.11 and Corollary 2.10. Let us assume $\gamma>0$. We first consider the following nonlinear eigenvalue problems:

$$
\alpha J_{n}=\frac{J_{2 n} J_{2 n+1}}{J_{2 n}+J_{2 n+1}}, \quad n \in \mathbb{Z}_{+} .
$$

The above set of equations has an explicit solution.

Lemma 4.1. For any fixed $\gamma>0$, (4.1) with $\alpha=2^{-\gamma-1}$ has a solution:

where,

$$
J_{n}=J_{n}^{*}, \quad n \in \mathbb{Z}_{+},
$$

$$
\begin{aligned}
J_{n}^{*} & =\{\alpha /(1-\alpha)\}(2 \alpha)^{[\log n / \log 2]}, \quad n \geqq 1, \\
& =1, \quad n=0,
\end{aligned}
$$

and for $x \in \mathbb{R},[x]$ is the largest integer $k$ satisfying $k \leqq x$.

Proof. Straightforward calculation proves the statement. 
Next we consider the following set of equations:

$$
\beta g_{n}=g_{2 n}+\frac{J_{2 n-1}^{*}}{J_{2 n-2}^{*}+J_{2 n-1}^{*}} g_{2 n-1}+\frac{J_{2 n}^{*}}{J_{2 n}^{*}+J_{2 n+1}^{*}} g_{2 n+1}, \quad n \in \mathbb{Z}_{+},
$$

where $g_{-1} \equiv 0$.

Lemma 4.2. Equation (4.3) with $\beta=2$ has a solution:

where,

$$
g_{n}=g_{n}^{*}, \quad n \in \mathbb{Z}_{+},
$$

$$
\begin{aligned}
g_{n}^{*} & =1, \quad n \geqq 2, \\
& =3 /\{2(2-\alpha)\}, \quad n=1, \\
& =3(1-\alpha) /\{2(2-\alpha)\}, \quad n=0 .
\end{aligned}
$$

Proof. Straightforward calculation proves that (4.4) satisfies (4.3).

We put $\alpha=2^{-\gamma-1}$ and $\beta=2$ in the following. We define the family $\left(J^{*}(t), t g^{*}(t)\right)$, $t>0$, of ferromagnetic pairs on $\mathbb{Z}_{+}$by

$$
\begin{aligned}
J^{*}(t)_{n}= & \frac{1}{\alpha} \frac{J_{2 n}^{*} J_{2 n+1}^{*}}{t g_{2 n+1}^{*}+J_{2 n}^{*}+J_{2 n+1}^{*}}, \quad n \in \mathbb{Z}_{+}, \\
g^{*}(t)_{n}= & \frac{1}{\beta}\left\{g_{2 n}^{*}+\frac{J_{2 n-1}^{*}}{t g_{2 n-1}^{*}+J_{2 n-2}^{*}+J_{2 n-1}^{*}} g_{2 n-1}^{*}\right. \\
& \left.+\frac{J_{2 n}^{*}}{t g_{2 n+1}^{*}+J_{2 n}^{*}+J_{2 n+1}^{*}} g_{2 n+1}^{*}\right\}, \quad n \in \mathbb{Z}_{+} .
\end{aligned}
$$

Then (3.3) implies

$$
f\left(J^{*}, g^{*}\right)=f\left(\alpha J^{*}(t), \beta \operatorname{tg}{ }^{*}(t)\right), \quad \text { for } t>0 .
$$

The following uniform estimates are easily derived by explicit calculations: For $n \in \mathbb{Z}_{+}$and $t>0$,

$$
\begin{gathered}
C_{4} n^{-\gamma}<J_{n}^{*}<C_{5} n^{-\gamma}, \quad n \geqq 1, \\
2 \alpha J_{n}^{*} \leqq J_{2 n}^{*}, \\
0<J_{n}^{*}-J^{*}(t)_{n}<C_{1} t, \\
0<g_{n}^{*}-g^{*}(t)_{n}<C_{2}, \\
\beta g^{*}(t)_{n}>g_{2 n}^{*},
\end{gathered}
$$

where $C_{i},(i=1,2,4,5)$ are positive constants independent of $n \in \mathbb{Z}_{+}$and $t>0$.

Next we define, for $t>0$,

$$
h_{1}(t)=\frac{f\left(\alpha J^{*}(t), \beta \operatorname{tg}{ }^{*}(t)\right)}{f\left(\alpha J^{*}, \beta \operatorname{tg}{ }^{*}(t)\right)}
$$

and

$$
h_{2}(t)=\frac{f\left(\alpha J^{*}, \beta \operatorname{tg}{ }^{*}(t)\right)}{f\left(\alpha J^{*}, \beta \operatorname{tg}{ }^{*}\right)}
$$


If we can show $h_{1}(t), h_{2}(t) \rightarrow 1$ as $t \downarrow 0$, then (3.1) with $\alpha=2^{-\gamma-1}$ and $\beta=2$ follows, and hence we have the value of $\tilde{d}\left(J^{*}\right)$.

Lemma 4.3. Suppose that we have the estimate:

$$
\varlimsup_{t \downarrow 0} \frac{\log f\left(J^{*}, t g^{*}\right)}{\log t} \leqq-\delta
$$

for some $\delta>1-1 / \gamma$. Then it holds that

$$
\lim _{t \downarrow 0} h_{i}(t)=1, \quad i=1,2,
$$

which, (as we remarked above,) implies

$$
\tilde{d}\left(J^{*}\right)=2 /(\gamma+2)
$$

Proof. First we note that

$$
J_{n}^{*} / J^{*}(t)_{n}-1=t /\left(4 \alpha J_{n}^{*}\right), \quad \text { if } \quad n>0 .
$$

Let $\varepsilon$ be an arbitrary constant satisfying $0<\varepsilon<1$. For sufficiently small $t>0$, we can define,

$$
N(t, \varepsilon)=\max \left\{N \in \mathbb{Z}_{+} \mid J^{*}(t)_{n} / J_{n}^{*}>1-\varepsilon \quad \text { if } \quad 0 \leqq n \leqq N\right\} .
$$

(Since we assumed $\gamma>0$, we have $N(t, \varepsilon)<\infty$.)

Let us show that if $t>0$ is sufficiently small,

$$
N(t, \varepsilon)^{\gamma} t>C_{3} \varepsilon,
$$

where $C_{3}$ is a positive constant independent of $t$ and $\varepsilon$. We have, with $N=N(t, \varepsilon)$ :

$$
\begin{aligned}
N(t, \varepsilon)^{\gamma} t & =N(t, \varepsilon)^{\gamma} 4 \alpha J_{N+1}^{*}\left(J_{N+1}^{*} / J^{*}(t)_{N+1}-1\right)>N(t, \varepsilon)^{\gamma} 4 \alpha J_{N+1}^{*} \varepsilon \\
& >4 \alpha C_{4} N(t, \varepsilon)^{\gamma}(N(t, \varepsilon)+1)^{-\gamma} \varepsilon>C_{3} \varepsilon,
\end{aligned}
$$

where we used (4.15), $J^{*}(t)_{N+1} / J_{N+1}^{*} \leqq 1-\varepsilon$, and (4.8). Thus we obtain (4.16).

We also see from the definition of $N(t, \varepsilon)$ that if $0 \leqq n \leqq N(t, \varepsilon)$,

$$
0<1-J^{*}(t)_{n} / J_{n}^{*}<\varepsilon,
$$

and from (4.3), (4.6), (4.1), (4.5), $J_{2 n}^{*} \geqq J_{2 n+1}^{*},(4.9)$, and (4.17):

$$
\begin{aligned}
0 & <g_{n}^{*}-g^{*}(t)_{n} \leqq \frac{\alpha}{\beta}\left\{\frac{J_{n-1}^{*}-J^{*}(t)_{n-1}}{J_{2 n-2}^{*}} g_{2 n-1}^{*}+\frac{J_{n}^{*}-J^{*}(t)_{n}}{J_{2 n}^{*}} g_{2 n+1}^{*}\right\} \\
& <\frac{1}{\beta} \varepsilon \frac{1}{2}\left(g_{2 n-1}^{*}+g_{2 n+1}^{*}\right)<\varepsilon / 2 .
\end{aligned}
$$

Next we decompose $h_{1}(t)$ :

$$
\begin{aligned}
h_{1}(t) & =\frac{f\left(\alpha J^{*}(t), \beta t g^{*}(t)\right)}{f\left(\alpha \widetilde{J}(t), \beta t g^{*}(t)\right)} \frac{f\left(\alpha \widetilde{J}(t), \beta \operatorname{tg}{ }^{*}(t)\right)}{f\left(\alpha J^{*}, \beta t g^{*}(t)\right)} \\
& \equiv P_{1}(t) Q_{1}(t),
\end{aligned}
$$


where

$$
\begin{aligned}
\widetilde{J}(t)_{n} & =J^{*}(t)_{n}, \quad n \leqq N(t, \varepsilon), \\
& =J_{n}^{*}, \quad n>N(t, \varepsilon) .
\end{aligned}
$$

Let us estimate $P_{1}(t)$. From (2.10) and (4.13) we have

$$
f\left(\alpha J^{*}, \beta t g^{*}\right) \geqq \alpha^{\delta-\varepsilon-1} \beta^{-\delta+\varepsilon} t^{-\delta+\varepsilon}
$$

if $t(>0)$ is small enough. Using (2.23), (2.22), and (4.10), we have:

$$
\left|\log P_{1}(t)\right| \leqq \frac{C_{7}}{N(t, \varepsilon) t f\left(\alpha J^{*}, t \beta g^{*}\right)},
$$

where $C_{7}$ is a positive constant independent of $t$ and $\varepsilon$. Furthermore, by the help of (4.20) and (4.16), we obtain

$$
\left|\log P_{1}(t)\right| \leqq C_{8}(\varepsilon) t^{1 / \gamma-1+\delta-\varepsilon},
$$

where $C_{8}(\varepsilon)$ is a positive constant independent of $t$. Therefore, if $\delta>1-1 / \gamma$, we have $P_{1}(t) \rightarrow 1$ as $t \downarrow 0$ by choosing sufficiently small $\varepsilon$. Let us estimate $Q_{1}(t)$. Since (4.17) implies

$$
(1-\varepsilon) J_{n}^{*}<\tilde{J}(t)_{n}, \quad n \in \mathbb{Z}_{+},
$$

we have

$$
1 \leqq Q_{1}(t)<\frac{f\left(\alpha(1-\varepsilon) J^{*}, \beta t g^{*}(t)\right)}{f\left(\alpha J^{*}, \beta t g^{*}(t)\right)}=\frac{1}{1-\varepsilon} \frac{f\left(\alpha J^{*},(1-\varepsilon)^{-1} \beta t g^{*}(t)\right)}{f\left(\alpha J^{*}, \beta t g^{*}(t)\right)} \leqq \frac{1}{1-\varepsilon},
$$

where we also used (2.22), (2.10) and (2.18). Thus we see that

$$
1 \leqq \lim _{t \downarrow 0} h_{1}(t) \leqq \varlimsup_{t \downarrow 0} h_{1}(t) \leqq(1-\varepsilon)^{-1}
$$

holds for any $\varepsilon<0$ sufficiently small. This proves $h_{1}(t) \rightarrow 1$ as $t \downarrow 0$.

The proof of $h_{2}(t) \rightarrow 1$ as $t \downarrow 0$ goes along the same line. We put

$$
\begin{aligned}
\tilde{g}(t)_{n} & =g^{*}(t)_{n}, \quad n \leqq N(t, \varepsilon), \\
& =g_{n}^{*}, \quad n>N(t, \varepsilon),
\end{aligned}
$$

and decompose $h_{2}(t)$ as

$$
\begin{aligned}
h_{2}(t) & =\frac{f\left(\alpha J^{*}, \beta t g^{*}(t)\right)}{f\left(\alpha J^{*}, \beta t \tilde{g}(t)\right)} \frac{f\left(\alpha J^{*}, \beta t \tilde{g}(t)\right)}{f\left(\alpha J^{*}, \beta t g^{*}\right)} \\
& \equiv P_{2}(t) Q_{2}(t) .
\end{aligned}
$$

Equations (2.19) and (2.18) together with (4.11) and (4.12) imply

$$
\left|\log P_{2}(t)\right| \leqq \frac{C_{10}}{N(t, \varepsilon) t f\left(\alpha J^{*}, t \beta g^{*}\right)},
$$

which, together with (4.20) and (4.16), yields

$$
\left|\log P_{2}(t)\right| \leqq C_{11}(\varepsilon) t^{1 / \gamma-1+\delta-\varepsilon}
$$


where $C_{11}(\varepsilon)$ is a positive constant independent of $t$. On the other hand, since (4.18) and (4.4) imply

$$
(1-\varepsilon) g_{n}^{*} \leqq \tilde{g}(t)_{n}, \quad n \in \mathbb{Z}_{+},
$$

we have

$$
1 \leqq Q_{2}(t) \leqq \frac{f\left(\alpha J^{*}, \beta t(1-\varepsilon) g^{*}\right)}{f\left(\alpha J^{*}, \beta t g^{*}\right)}=\frac{1}{1-\varepsilon} \frac{f\left((1-\varepsilon)^{-1} \alpha J^{*}, \beta t g^{*}\right)}{f\left(\alpha J^{*}, \beta t g^{*}\right)} \leqq \frac{1}{1-\varepsilon},
$$

where we used (2.19), (2.10) and (2.22). This proves $h_{2}(t) \rightarrow 1$ as $t \downarrow 0$.

Let us continue the proof of the theorem. For each $\gamma>0$, we denote by $J_{\gamma}$, the interaction $J^{*}$ defined by (4.2) with $\alpha=2^{-\gamma-1}$, and similarly, we define $g_{\gamma}$. We have proved that, if there exists a constant $\delta>1-1 / \gamma$ that satisfies

$$
\varlimsup_{t \downarrow 0} \frac{\log f\left(J_{\gamma}, t g_{\gamma}\right)}{\log t} \leqq-\delta
$$

then we have

$$
\tilde{d}\left(J_{\gamma}\right)=2 /(\gamma+2) \text {. }
$$

Since, from (2.26), we have the trivial estimate (4.21) with $\delta=0,(4.22)$ holds for $0<\gamma<1$, in particular, for $0<\gamma \leqq 1 / 2$. Suppose that we have proved (4.22) for $\gamma \leqq \tilde{\gamma}$. Then for any $\gamma$ satisfying $\tilde{\gamma}<\gamma$, we have:

$$
\varlimsup_{t \downarrow 0} \frac{\log f\left(J_{\gamma}, t g_{\gamma}\right)}{\log t} \leqq \varlimsup_{t \downarrow 0} \frac{\log f\left(J_{\tilde{\gamma}}, t g_{\gamma}\right)}{\log t}=\tilde{d}\left(J_{\tilde{\gamma}}\right) / 2-1=-(\tilde{\gamma}+1) /(\tilde{\gamma}+2) .
$$

Therefore (4.21) with $\delta=(\tilde{\gamma}+1) /(\tilde{\gamma}+2)$ holds. Since, for $\gamma \leqq \tilde{\gamma}+1$,

$$
1-1 / \gamma \leqq 1-1 /(\tilde{\gamma}+1)=\tilde{\gamma} /(\tilde{\gamma}+1)<(\tilde{\gamma}+1) /(\tilde{\gamma}+2)=\delta,
$$

(4.22) holds for $\tilde{\gamma}<\gamma \leqq \tilde{\gamma}+1$. Therefore we have (4.22) for all $\gamma>0$ by induction.

For any $J$ satisfying (1.5), applying (2.24), we have $\tilde{d}(J)=\tilde{d}\left(J_{\gamma}\right)$. This completes the proof.

\section{Appendix}

For a ferromagnetic pair $(J, g)$, we prove

that is,

$$
\left\langle\phi_{0}^{2}\right\rangle(J, g)=\frac{1}{g_{0}}+\frac{1}{J_{0}^{-1}}+\frac{1}{g_{1}}+\frac{1}{J_{1}^{-1}}+\cdots,
$$

where

$$
\left\langle\phi_{0}^{2}\right\rangle(J, g)=\lim _{n \rightarrow \infty} f_{n},
$$

$$
\begin{aligned}
f_{2 n-1} & =\frac{1}{g_{0}}+\frac{1}{J_{0}^{-1}}+\frac{1}{g_{1}}+\frac{1}{J_{1}^{-1}}+\cdots+\frac{1}{g_{n}}, \quad n>0, \\
f_{2 n} & =\frac{1}{g_{0}}+\frac{1}{J_{0}^{-1}}+\frac{1}{g_{1}}+\frac{1}{J_{1}^{-1}}+\cdots+\frac{1}{g_{n}}+\frac{1}{J_{n}^{-1}}, \quad n>0 .
\end{aligned}
$$


Since Seidel-Stern's theorem ([2] p. 87) implies (because of $\left.\sum_{n \in \mathbb{Z}_{+}}\left(g_{n}+J_{n}^{-1}\right)=\infty\right)$

$$
\lim _{n \rightarrow \infty} f_{2 n}=\lim _{n \rightarrow \infty} f_{2 n-1}
$$

it suffices to show that

$$
\left\langle\phi_{0}^{2}\right\rangle(J, g)=\lim _{N \rightarrow \infty} f_{2 N} .
$$

From

Put $\mu_{0}=g_{0}+J_{0}$ and $\mu_{n}=g_{n}+J_{n-1}+J_{n}, n>0$.

$$
\left\langle\phi_{0} \phi_{n}\right\rangle(J, g)=(D(g)-H(J))_{0 n}^{-1}
$$

we have

$$
\begin{gathered}
\mu_{0}\left\langle\phi_{0}^{2}\right\rangle(J, g)-J_{0}\left\langle\phi_{0} \phi_{1}\right\rangle(J, g)=1, \\
\mu_{n}\left\langle\phi_{0} \phi_{n}\right\rangle(J, g)-J_{n-1}\left\langle\phi_{0} \phi_{n-1}\right\rangle(J, g)-J_{n}\left\langle\phi_{0} \phi_{n+1}\right\rangle(J, g)=0, \quad n \geqq 1 .
\end{gathered}
$$

We modify the original ferromagnetic pair $(J, g)$. Fix $N>0$. Put $J_{N}=0$ and increase $g_{N}$ so that the value of $\mu_{N}$ does not change. Write the resulting ferromagnetic pair as $(\tilde{J}, \tilde{g})$. Put

$$
c_{n}=J_{n-1}\left[1-\left\langle\phi_{0} \phi_{n}\right\rangle(\tilde{J}, \tilde{g}) /\left\langle\phi_{0} \phi_{n-1}\right\rangle(\tilde{J}, \tilde{g})\right], \quad n>0 .
$$

Then the analog of (A.5) and (A.6) imply

$$
\begin{aligned}
f(\tilde{J}, \tilde{g}) & =1 /\left(g_{0}+c_{1}\right), \\
c_{n} & =1 /\left\{1 / J_{n-1}+1 /\left(g_{n}+c_{n+1}\right)\right\}, \quad N>n>0, \\
c_{N} & =1 /\left\{1 / J_{N-1}+1 /\left(g_{N}+J_{N}\right)\right\} .
\end{aligned}
$$

As is easily seen from (A.7), (A.8), (A.9), and (A.1), it holds that

$$
f(\tilde{J}, \tilde{g})=f_{2 N}
$$

On the other hand, from the definition of $(\tilde{J}, \tilde{g}), f(\tilde{J}, \tilde{g})$ can be written in the form of (2.8) that is a finite volume approximation of the original expression (2.8): i.e., the summation is now taken over all walks in $W(0,0)$ not passing through the point $N+1$. Since the finite volume approximation of $(2.8)$ converges to $f(J, g)=$ $\left\langle\phi_{0}^{2}\right\rangle(J, g)$ in the limit $N \rightarrow \infty$, we have (A.3).

Acknowledgements. We would like to thank Professor M. Miyamoto for taking interest in our work, and for informing us of his work before publication.

\section{References}

1. Ma, S. K.: Modern theory of critical phenomena. New York: W. A. Benjamin 1976

2. Jones, W. B., Thron, W. J.: Continued fractions, analytic theory and applications. London: Addison-Wesley 1980

3. Rammal, R., Toulouse, G.: Random walks on fractal structures and percolation clusters. J. Phys. Lett. 44, L13-22 (1983) 
4. Ito, K., McKean, H. P.: Diffusion processes and their sample paths. Berlin: Heidelberg, New York: Springer 1965

5. Kasahara, Y.: Spectral theory of generalized second order differential operators and its applications to Markov processes. Jpn J. Math. 1, 67-84 (1975)

6. Hattori, K., Hattori, T., Watanabe, H.: Gaussian field theories on general networks and the spectral dimensions. To appear in Progr. Theor. Phys. Suppl. 92, (1987)

7. Fujita, T.: A fractional dimension, self-similarity and a generalized diffusion operator. To appear in The Proceeding of Katata Symposium

8. Miyamoto, M.: Private communication

Communicated by H. Araki

Received August 16, 1986 